\title{
Malaria epidemiological research in the Republic of Congo
}

\author{
Felix Koukouikila-Koussounda ${ }^{1}$ and Francine Ntoumi $i^{1,2,3^{*}}$
}

\begin{abstract}
Background: Reliable and comprehensive information on the burden of malaria is critical for guiding national and international efforts in malaria control. The purpose of this review is to provide an overview of published data and available information on malaria resulting from field studies/investigations conducted in the Republic of Congo (RoC) from 1992 to 2015, as baseline for assisting public health authorities and researchers to define future research priorities as well as interventions.

Methods: This review considers data from peer-reviewed articles and information from the National Malaria Control Programme reports, based on field investigations or samples collected from 1992 to 2015. Peer-reviewed papers were searched throughout online bibliographic databases PubMed, HINARI and Google Scholar using the following terms: "malaria”, "Congo", "Brazzaville", "prevalence”, "antimalarial", "efficacy", "falciparum”, "'genetic", "diversity". Original articles and reviews were included and selection of relevant papers was made.

Results: Twenty-eight published articles were included in this review and two additional records from the National Malaria Control Programme were also considered. The majority of studies were conducted in Brazzaville and Pointe-Noire.

Conclusion: The present systematic review reveals that number of studies have been conducted in the RoC with regard to malaria. However, their results cannot formally be generalized at the country level. This suggests a need for implementing regular multisite investigations and surveys that may be representative of the country, calling for the support and lead of the Ministry of Health.
\end{abstract}

Keywords: Malaria, Plasmodium falciparum, Review, Health research, Republic of Congo

\section{Background}

Malaria is a life-threatening disease caused by protozoan parasites of the genus Plasmodium that are transmitted to humans through the bites of infected Anopheles mosquitoes. Five different Plasmodium species have been demonstrated to infect humans: Plasmodium falciparum, Plasmodium vivax, Plasmodium ovale, Plasmodium malariae and Plasmodium knowlesi. Of these, P. falciparum is the most dangerous, with the highest rates of complications and mortality [1].

The scaling-up of interventions has reduced the number of malaria cases and deaths between 2000 and 2015

\footnotetext{
*Correspondence: fntoumi@fcrm-congo.com

1 Fondation Congolaise Pour la Recherche Médicale, Villa D6, WHO AFRO

Campus, Brazzaville, Republic of Congo

Full list of author information is available at the end of the article
}

[2-4]. In 2000, the World Health Organization (WHO) estimated 262 million cases of malaria globally, leading to 839,000 deaths against 214 million cases and 438,000 deaths in 2015 [2]. Sub-Saharan Africa remains the region with the highest disease burden and accounts for 88 and $90 \%$ of the global clinical cases and deaths, respectively [2].

The Republic of Congo ( $\mathrm{RoC}$ ) is one of the 54 countries where malaria transmission is still high [2]. The country is located in the central-western part of sub-Saharan Africa, along the Equator, laying between latitudes $4^{\circ} \mathrm{N}$ and $5^{\circ} \mathrm{S}$, and longitude $11^{\circ}$ and $19^{\circ} \mathrm{E}$ (Fig. 1). It occupies a total surface of $342,000 \mathrm{~km}^{2}$ with a population estimated to be $4,800,000$ inhabitants, with $61 \%$ of its total population living in the two biggest cities, namely Brazzaville and Pointe-Noire [5]. Accordingly, the RoC is one of the 


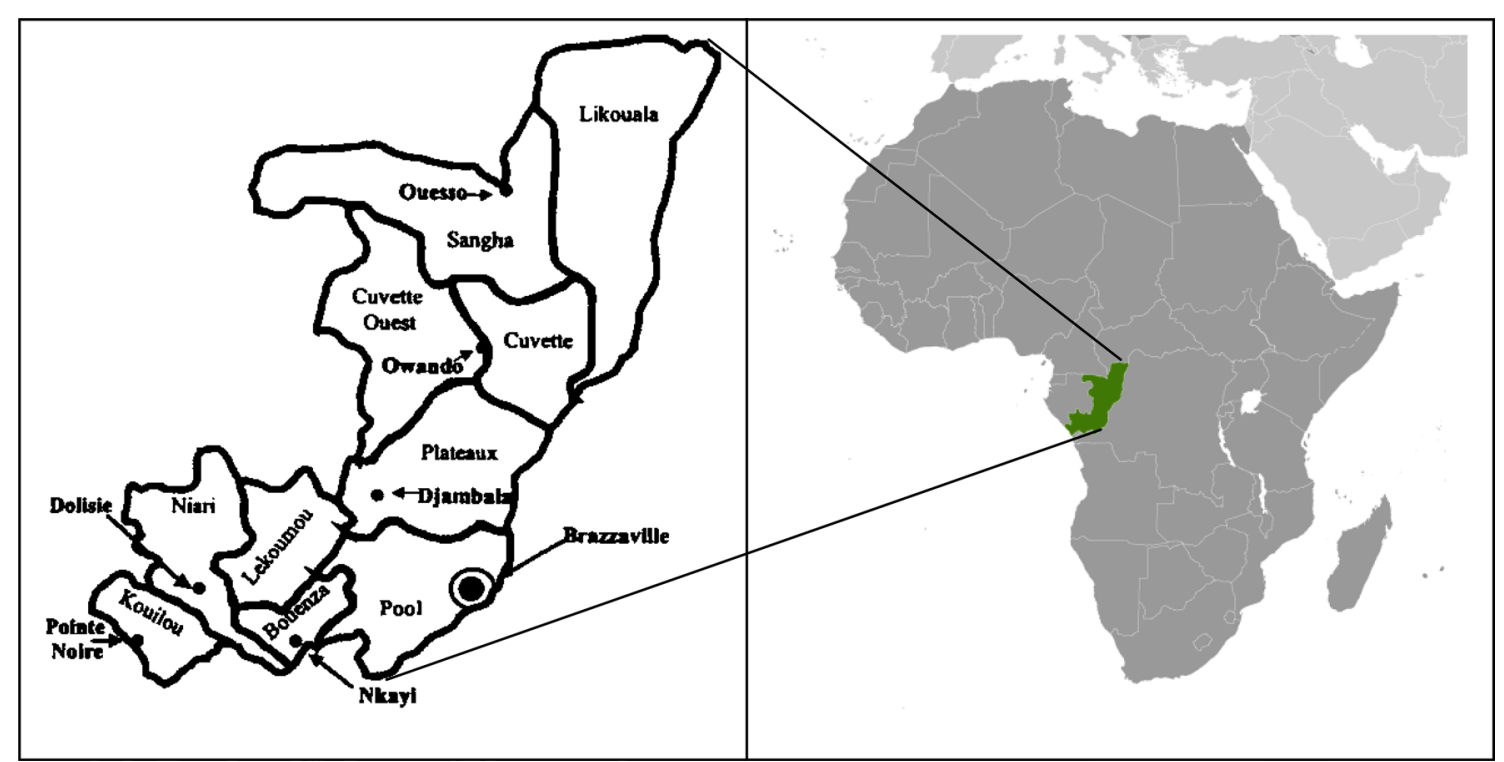

Fig. 1 Map of the Republic of Congo (RoC) with its 12 departments

most urbanized countries in Africa. The capital, Brazzaville, is located along the Congo River, in the south of the country, immediately across from Kinshasa, the capital of the Democratic Republic of Congo. Since the country is located on the Equator, the climate is consistently humid year-round, with the average day temperature of $25^{\circ} \mathrm{C}$ and night between 16 and $21^{\circ} \mathrm{C}$ [6]. Two-thirds and one-third of the surface area of $\mathrm{RoC}$ is covered by forests and savannah, respectively. The average yearly rainfall ranges from $1100 \mathrm{~mm}$ in the south to over $2000 \mathrm{~mm}$ in the central and north parts of the country. The rainy season which lasts 9 months, has two rainfall maxima: one in March-May and another in September-November [6]. The dry season is from June to August. A recent entomological survey in $\mathrm{RoC}$ jointly conducted by the WHO and the Ministry of Health and Population (MHP) showed that the transmission dynamic of malaria in the country follows two different patterns: (1) a year-round perennial transmission in forest areas, with an estimated entomological inoculation rate (EIR) of 200-1000 infective bite/person/year, and (2) a seasonal transmission in savanna areas where the high transmission period lasts 7-10 months and is directly correlated with the rainfall and the EIR is estimated to be $80-200$ infective bites/ person/year [7]. Despite considerable efforts and progress in malaria control over the past decades [adoption of artemisinin-based combination therapy (ACT) for the treatment of uncomplicated malaria in 2006, using either artemether-lumefantrine (AL) or artesunate-amodiaquine (ASAQ), use of intermittent preventive treatment with sulfadoxine-pyrimethamine for pregnant women
(IPTp-SP), mass distribution of long-lasting insecticidetreated mosquito nets (LLINs) from 2008 to 2012 and free anti-malarial treatment for children aged $>15$ years since 2008], it remains one of the important public health problems [8]. All over the country, P. falciparum is the predominant malaria parasite and Anopheles gambiae the predominant mosquito vector. The latest estimations from the National Malaria Control Programme (NMCP) indicate that clinical malaria account for $47.9 \%$ of all outpatient consultations in public hospitals, $64.8 \%$ of hospital admissions and $18.4 \%$ of deaths [5].

Clearly, the country is still struggling with the control phase where expensiveness of the control program as well as its sustainability, resistance of the parasite to anti-malarial drugs and that of vectors to insecticides are some of the challenges. However, some research publications have reported a decrease in clinical malaria prevalence in Southern and Northern sentinel sites of the country $[9,10]$.

The purpose of this review was to provide an overview of published data and available information from the NMCP of the MHP on malaria situation in RoC including prevalence/incidence trends, vectors, anti-malarial drug efficacy and parasite genetics based on field studies conducted from 1992 to 2015 . This may assist in defining future research and intervention priorities.

\section{Methods}

\section{Search strategy}

To collect research data on malaria in $\mathrm{RoC}$, peerreviewed articles have been retrieved from online 
bibliographic databases PubMed, HINARI and Google scholar using the following keywords: "malaria", "Congo", "Brazzaville", "1992", "prevalence", "antimalarial", "efficacy”, "falciparum", "genetic", "diversity", "resistance”, "markers". Reference lists of selected papers were used as leads for identification of additional studies. The Boolean operators "AND", "OR" and ">" were used to combine two or three terms. In addition, reports from the NMCP were reviewed at the NMCP headquarters in Brazzaville. Predefined medical subject heading $(\mathrm{MeSH})$ was not used to avoid restricting searches.

\section{Study/document selection and data consideration}

Studies were included in the review if they explicitly reported on one of the considered aspect of malaria in RoC with: (1) samples collected in the country, (2) a clear description of the methods section (providing the following information: study area, period of sample collection, type of samples collected and study population) and (3) no review articles. An overall of 33 studies were retrieved from the search of peer-reviewed papers, of which five were excluded because of duplications (similar analysis on same samples or study population) or they were review articles. Therefore, 28 studies including full text articles, and short reports written in English or in French were selected and reviewed. Two additional documents from the NMCP were considered (Fig. 2).

\section{Results}

\section{Malaria parasite prevalence}

Published articles included in this review were of samples collected from 1992 to 2015 (Table 1). Of these, nine articles were identified with information on the incidence or the prevalence of malaria parasite infection (Table 2) $[9,11-18]$. Most of these studies were performed in

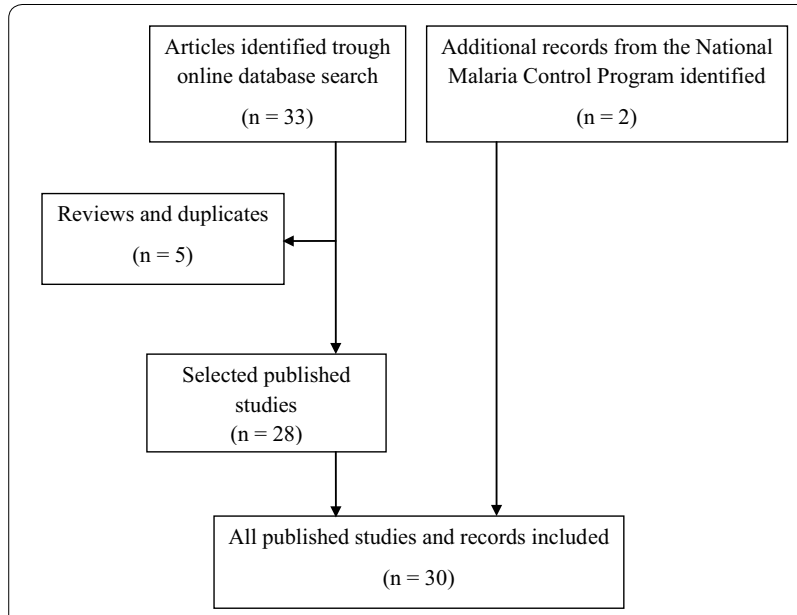

Fig. 2 Summary of the search strategy children aged $\leq 15$ years. Investigations were conducted in different areas of the country, with most of them being located in Brazzaville and mainly reported the presence of one malaria species, namely $P$. falciparum. There was only one study in which three plasmodial species were detected with a predominance of $P$. falciparum over $P$. malariae (2.9\%) and P. ovale (0.7\%) [11]. Six of the P. ovale isolates (two isolates from Brazzaville, two from PointeNoire and two from Gamboma) were further characterized by Oguiket et al. using conventional and real-time quantitative PCR methods to discriminate $P$. ovale curtisi and $P$. ovale wallikeri. The two isolates from Brazzaville were identified as $P$. ovale curtisi, while the remaining four isolates were identified as $P$. ovale wallikeri [19]. In another study, a focus was put on providing evidence of transmission of $P$. vivax in RoC [12]. The authors found that $13 \%$ of samples, collected in 2007 in Pointe-Noire and tested by enzyme-linked immunosorbent assay, had antibodies to $P$. vivax-specific antigens. Accordingly, they concluded that in conjunction with the frequent reports of travellers returning from western and central Africa with diagnosed $P$. vivax infections, their findings make an argument for the presence and continued transmission of $P$. vivax in RoC. How and where precisely is still unclear and deserves to be further investigated.

The study with the largest sample size was conducted from 2003 to 2006 in southern part of Brazzaville just before introduction of ACT in the country [13]. The authors found that in peri-urban area, of more than 1090 febrile patients examined, $44.7 \%$ had clinical malaria. Whereas in urban area, of 10,603 febrile patients examined, only $23.8 \%$ had clinical malaria. It was also observed that the pick of infections occurred between November and January and in March-April [13]. In the study conducted by Tsumori et al. [11] with samples collected in 2005-2006 from patients residing in urban and periurban areas of Brazzaville, Pointe Noire and Gamboma, plasmodial infection was screened by microscopy and by PCR. By microscopy, 37\% of patients residing in urban areas were positive for P. falciparum compared to 59\% of those residing in the peri-urban areas. With regard to PCR corrected results, the percentages rose to $42 \%$ P. falciparum positive for the urban residents and $75 \%$ positive for peri-urban residents, indicative a percentage of 11 and $20 \%$ of submicroscopic infections in these two populations, respectively. Another important study conducted before adoption of ACT was concomitantly done in different pediatric services of four hospitals in Brazzaville from January to August 2006 [14]. With the objective of assessing the real prevalence of severe malaria among young children in pediatric services, this study included more than 10,000 children. The overall severe malaria prevalence was $14.7 \%$. Children of $>5$ years old 
Table 1 Summary of published articles included in the review

\begin{tabular}{|c|c|c|c|c|}
\hline References & Period of sample collection & Year of publication & Study area & Age of participants \\
\hline Chandenier et al. [20] & 1993 & 1995 & Brazzaville, Niari, Kouilou, Pool & $6-10$ years \\
\hline Durand et al. [21] & 1998 & 2003 & Pointe-Noire & No limit \\
\hline Nsimba et al. [22] & 1999-2002 & 2004 & Brazzaville, Pointe-Noire & $0.5-<5$ years \\
\hline Nsimba et al. [23] & 1999-2002 & 2005 & Brazzaville, Pointe-Noire & $0.5-<5$ years \\
\hline Mayengue et al. [24] & 2003 & 2005 & Brazzaville & $\leq 5$ years \\
\hline Ndounga et al. [25] & 2003-2004 & 2007 & Brazzaville & $<5$ years \\
\hline Ndounga et al. [13] & $2003-2006$ & 2008 & Brazzaville & No limit \\
\hline Van den Broek et al. [26] & 2004 & 2006 & Kindamba & $0.5-<5$ years \\
\hline Mayengue et al. [27] & 2005 & 2011 & Brazzaville & No limit \\
\hline Ndounga et al. [28] & 2005 & 2013 & Brazzaville & No limit \\
\hline Pradine et al. [29] & $2005-2006$ & 2006 & Pointe-Noire & $1.4-17$ years \\
\hline Tsumori et al. [11] & 2005-2006 & 2011 & Brazzaville, Gamboma, Pointe-Noire & No limit \\
\hline Oguike et al. [19] & 2005-2006 & 2011 & Brazzaville, Gamboma, Pointe-Noire & No limit \\
\hline Moyen et al. [14] & 2006 & 2010 & Brazzaville & $\leq 15$ years \\
\hline Ndounga et al. [30] & 2006 & 2012 & Brazzaville & $0.5-10$ years \\
\hline Murai et al. [31] & 2006 & 2015 & Brazzaville, Gamboma, Pointe-Noire & No limit \\
\hline Mita et al. [32] & 2006 & 2016 & Brazzaville, Gamboma, Pointe-Noire & No limit \\
\hline Culleton et al. [12] & 2007 & 2009 & Pointe-Noire & No limit \\
\hline Koekemoer et al. [33] & 2009 & 2011 & Boutoto & Not applicable \\
\hline Koukouikila-Koussounda et al. [15] & 2010 & 2012 & Brazzaville & $<10$ years \\
\hline Ibara-Okabande et al. [34] & 2010-2011 & 2012 & Brazzaville & $<10$ years \\
\hline Ndounga et al. [35] & 2010-2011 & 2015 & Brazzaville & $<10$ years \\
\hline Ossou-Nguiet et al. [18] & 2011 & 2013 & Brazzaville & $0.4-14$ years \\
\hline Mbongo et al. [17] & $2011-2013$ & 2015 & Brazzaville & $15-39$ years \\
\hline Ntoumi et al. [9] & $2011-2012$ & 2013 & Brazzaville, Pointe-Noire & $\leq 15$ years \\
\hline Koukouikila-Koussounda et al. [36] & $2012-2013$ & 2015 & Brazzaville & $12-44$ years \\
\hline Ntoumi et al. [16] & $2012-2013$ & 2016 & Brazzaville & $12-44$ years \\
\hline Singana et al. [10] & 2012-2013 & 2016 & Owando & $<12$ years \\
\hline
\end{tabular}

Table 2 Main findings of studies that assessed the prevalence of malaria parasite infection

\begin{tabular}{|c|c|c|c|c|c|c|c|}
\hline \multirow[t]{2}{*}{ References } & \multirow{2}{*}{$\begin{array}{l}\text { Period of sample } \\
\text { collection }\end{array}$} & \multirow{2}{*}{$\begin{array}{l}\text { Year of } \\
\text { publication }\end{array}$} & \multicolumn{2}{|c|}{ Asymptomatic infection } & \multicolumn{2}{|c|}{ Infection in febrile patients } & \multirow{2}{*}{$\begin{array}{l}\text { Severe malaria } \\
\text { Microscopy (\%) }\end{array}$} \\
\hline & & & Microscopy (\%) & PCR (\%) & Microscopy (\%) & PCR (\%) & \\
\hline Ndounga et al. [13] & 2003-2006 & 2008 & - & - & $23.8^{\mathrm{b}, \mathrm{e}}$ versus $44.7^{\mathrm{a}, \mathrm{e}}$ & - & - \\
\hline Tsumori et al. [11] & $2005-2006$ & 2011 & - & - & $37^{\mathrm{b}, \mathrm{e}}$ versus $59^{\mathrm{a}, \mathrm{e}}$ & $42^{\mathrm{b}, \mathrm{e}}$ versus $75^{\mathrm{a}, \mathrm{e}}$ & - \\
\hline Moyen et al. [14] & 2006 & 2010 & - & - & - & - & $14.7^{b}$ \\
\hline $\begin{array}{l}\text { Koukouikila-Kous- } \\
\text { sounda et al. [15] }\end{array}$ & 2010 & 2012 & $8.6^{\mathrm{a}, \mathrm{c}}$ & $16^{\mathrm{a}, \mathrm{c}}$ & - & - & - \\
\hline $\begin{array}{l}\text { Ossou-Nguiet et al. } \\
\quad[18]\end{array}$ & 2011 & 2013 & - & - & & - & $34.9^{b}$ \\
\hline Mbongo et al. [17] & $2011-2013$ & 2015 & $4.4^{b, d}$ & - & - & - & - \\
\hline Ntoumi et al. [9] & $2011-2012$ & 2013 & - & - & $12^{\mathrm{b}, \mathrm{c}}$ versus $17^{\mathrm{b}, \mathrm{d}}$ & - & - \\
\hline Ntoumi et al. [16] & 2012-2013 & 2016 & $7^{\mathrm{a}, \mathrm{d}}$ & $19^{\mathrm{a}, \mathrm{d}}$ & - & - & - \\
\hline
\end{tabular}

\footnotetext{
a Prevalence in sub-urban area

b Prevalence in urban areas

c Children

d Pregnant women

e No age limit
} 
were the most affected group as the rate reached $57.6 \%$. The authors also determined $26.3 \%$ of death rate in this children population [14].

Following the replacement of chloroquine with ASAQ or AL in 2006 for the treatment of uncomplicated malaria, the first study that reported the prevalence of malaria parasite infection was conducted in 2010 in a peri-urban area of southern Brazzaville [15], an area which has been characterized as highly endemic with perennial malaria transmission [37]. In this study, from April to June 2010, 313 children below 10 years of age enrolled in a cohort for malaria surveillance were screened for P. falciparum asymptomatic carriage using microscopy and PCR as diagnostic techniques. The reported prevalence of infection was 8.6 and $16 \%$ by microscopy and PCR, respectively [15]. In a prospective and longitudinal study conducted from January 2011 to December 2013 at University Hospital Centre of Brazzaville, 13,883 pregnant women were screened by microscopy at delivery for malaria parasite carriage and newborns from positive mothers were further screened. A total of 610 mothers (4.4\%) were found positive and $64 \%$ of newborns from these mothers also carried malaria parasites [17]. From October 2011 to February 2012, Ntoumi et al. carried out a malaria survey among under 15 years old children and pregnant women in different public health centers in Brazzaville and Pointe-Noire [9]. The main objective of the study was to document laboratory-confirmed cases of malaria using microscopy and/or rapid diagnostic tests (RDTs). P. falciparum was the only species detected and the prevalence of infections among more than 3000 children and 700 pregnant women ranged from 8 to 29 , and 8 to $24 \%$, respectively [9]. Another study by Ntoumi et al. using blood samples collected from March 2012 to December 2013 in an antenatal clinic located in periurban area of southern Brazzaville among pregnant women, reported a prevalence of asymptomatic $P$. falciparum infection at 7 and 19\% when using microscopy and PCR, respectively [16]. A study by Ossou-Nguiet et al. conducted from July to December 2011 at the intensive care department of pediatric service of the University Hospital Center focused on determining the frequency and determinants of severe malaria among hospitalized children. A total of 1135 children were enrolled in this study and $34.9 \%$ (396) of them were diagnosed as severe malaria cases. Among them, 35.9\% were further classified as coma cases, while $23.5,20.8,10.3,7.4$ and $3.6 \%$ were found to experience convulsions, coma and anaemia, prostration, convulsions and anaemia, and convulsions and respiratory distress, respectively [18].

Apart from the published articles, data available at the Ministry of Public Health and provided by the NMCP were also considered. In the national malaria report 2015 (released in 2016) [8] and the strategic plan for malaria control in the RoC from 2014 to 2018 (released in 2014) [5], the number of confirmed malaria cases recorded in public health centres countrywide from 2008 to 2015 were reported. Overall, malaria showed a fluctuation trend during the last 8 years (Fig. 3). The number of cases was 160,000 in $2008,150,000$ in $2009,260,000$ in $2010,277,263$ in $2011,117,640$ in $2012,182,026$ in 2013, 248,159 in 2014 and 198,047 in 2015 [5, 8]. As there is no such data for the years before ACT, it is difficult to give a comprehensive interpretation of this fluctuation.

\section{Malaria vectors}

Malaria entomological studies are very limited in the RoC. From 1992 to June 2016, only one published investigation was found [33]. Conducted in 2009 in the village Boutoto located in the vicinities of Pointe-Noire, the study aimed to obtain the baseline vector information. Anopheles gambiae sensu stricto (s.s.) was the only mosquito species identified, and it had a high $P$. falciparum infection rate $(9.6 \%)$. In this study, insecticide susceptibility was also assessed and the authors mentioned that multiple insecticide resistance was detected in this vector population with full susceptibility observed to only one insecticide class, the organophosphate [33].

In 2014, the NMCP documented entomological surveys conducted in 2013 by Antonio-Nkondjio and Bitsindu in the Bouenza department located at $300 \mathrm{~km}$ southern Brazzaville. Anopheles gambiae s.l. and Anopheles funestus were reported as the predominant vectors, while other species including Anopheles coustani, Anopheles hancocki, Anopheles nili, Anopheles moucheti and Anopheles paludis were found to be potential secondary vectors [5].

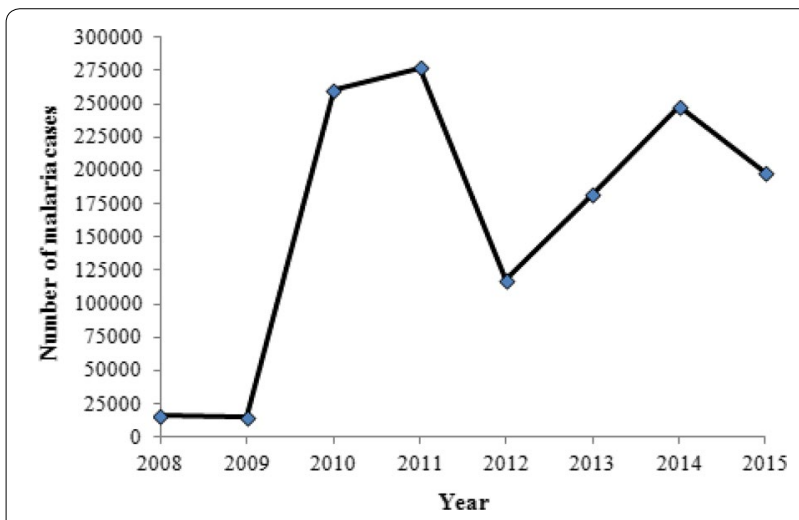

Fig. 3 Number of malaria cases as reported by the National Malaria Control Program 


\section{Genetic diversity and multiplicity of Plasmodium falciparum in isolates from RoC}

Number of information on the genetic diversity and the multiplicity of $P$. falciparum infections have been generated using samples from the RoC. A total of 8 published articles have been found, for which, different molecular markers have been used. The genes for the merozoite surface protein 1 and 2 have been used, $m s p 2$ alone has been used in four investigations $[9,15,16,34]$, msp1 and $m s p 2$ in one study [27], and microsatellites in two studies with one utilizing $m s p 1$ in addition to the microsatellite markers $[11,21]$.

In 2003, analysing 32 isolates of $P$. falciparum collected in 1998 at Pointe Noire, Durand et al. reported a high genetic diversity based on 28 microsatellites with a mean number of alleles per locus of 7.75 and the expected heterozygosity of 0.78 [21]. This trend was further confirmed by Tsoumori et al. who analysed microsatellite loci of $P$. falciparum isolates collected in urban and peri-urban areas in southern Brazzaville in 2005 [11]. In the study by Mayengue et al. [27], with 125 P. falciparum clinical isolates collected from children in south part of Brazzaville in 2005 , the allelic specific $m s p 1$ and $m s p 2$ genotyping showed that malaria parasite population in Brazzaville is highly diverse. A total of $15 m s p 1$ and $20 m s p 2$ distinct alleles were identified. For $m s p 1$, K1 was found to be the predominant allelic type, whereas 3D7 family was the most prevalent for $m s p 2$. The overall mean MOI was 2.2 and $72 \%$ of the isolates carried more than one genotype [27]. In 2010, Four years after adoption of ACT in the country, Koukouikila-Koussounda et al. characterized the genetic polymorphism of $m s p 2$ gene in P. falciparum isolates collected from Congolese children with asymptomatic infections [15]. Eighten different allele types were identified, suggestive of a high genetic diversity, in which, 8 belonged to the 3D7 family, which was found to be the predominant family, and 10 to FC27. However, the MOI and the rate of isolates with multiple genotypes were found to be low, 1.3 and $28 \%$, respectively [15]. The cohort of children enrolled in this study was followed up for a year to monitor uncomplicated falciparum malaria episodes and isolates collected from some of the children who experienced uncomplicated malaria were later on analysed by Ibara-Okabando et al. [34] with regard to parasite diversity using $m s p 2$ as molecular marker. A high genetic diversity of parasite population was observed with 21 different alleles detected (11 for 3D7 family and 10 for the FC27). Here, the MOI of 1.7 was determined and $54 \%$ the isolates harboured more than one $m s p 2$ genotype [34].

Recent studies among children and pregnant women from Brazzaville and Pointe-Noire conducted in 20112012 by Ntoumi et al. [9] provided additional information on the genetic diversity and the MOI. The molecular characterization of the $m s p 2$ gene revealed the presence of 11 (5 of 3D7 and 6 of FC27) and 22 alleles (13 of 3D7 and 9 of FC27) in P. falciparum isolates collected from 2011 to 2012 from children aged >15 years with uncomplicated malaria in Brazzaville and Pointe-Noire, respectively. The MOI was similar (about 1.7) in the two cities [9]. In another study, analysing isolates collected in 20122013 from pregnant women with asymptomatic P. falciparum infection using the same molecular marker, 11 alleles of the 3D7 family and 18 of the FC27 family were detected [16]. This is indicative of a higher genetic diversity in isolates from pregnant women that that of children. The rate of alleles belonging to the 3D7 and FC27 families was 62 and 38\%, respectively. The authors also reported that $40.3 \%$ of the isolates harboured more than one $m s p 2$ genotype and the overall MOI was 1.6 [16].

\section{Drug efficacy studies}

In the period 1992 to 2005, before adoption of ACTs, six efficacy in vivo studies had been conducted, of which one was based on the $1973 \mathrm{WHO}$ protocol for asymptomatic children to assess chloroquine efficacy [20], one on 14-day follow-up WHO protocol to assess chloroquine and sulfadoxine-pyrimethamine (SP) [22], and four on the current 28-day follow-up WHO protocol to evaluate therapeutic efficacy of chloroquine [24], SP [25], ASAQ, $\mathrm{AL}$ and AS + SP [26], AL and then ASAQ [28, 30]. The study based on the 1973 WHO protocol was conducted in 1993 in three southern regions of the country (Niari, Kouilou and Pool which included Brazzaville) and the authors reported that 7 days after the standard threeday treatment with chloroquine at $25 \mathrm{mg} / \mathrm{kg}, 20-60 \%$ of cases were still found to carry malaria parasites [20]. In the study using the 14-day follow-up WHO protocol and conducted in Brazzaville and Pointe-Noire from 1999 to 2002, the cure rate of chloroquine was $38.5 \%$ and that of SP was $95.8 \%$. SP efficacy was further assessed in the second phase of this study and the cure rate of $100 \%$ was recorded [22]. However, studies carried out using the 28-day follow-up WHO protocol showed high level of treatment failure for chloroquine (95.7\%) [24] and SP (31.2\%) [25], while AL and ASAQ were found to be highly effective to treat uncomplicated malaria with the reported 28-day PCR-corrected cure rates of 96.9 [30] and $100 \%$ [26] for AL, and 94.4\% [28] and 98.5\% [26] for ASAQ. AS + SP, with the 28-day PCR-corrected cure rate of $90 \%$ was also effective but less than AL and ASAQ [26].

In 2006, the government of the RoC adopted a new treatment policy for uncomplicated malaria with ASAQ and $\mathrm{AL}$ as the first and second-line drugs, respectively [38]. However, in 2014, AL became the first- and ASAQ the second-line drug. Since 2006, two studies were 
conducted, one in Brazzaville and the other in Owando (located at $550 \mathrm{~km}$ to north of Brazzaville), to evaluate the efficacy of ASAQ and AL. The study in Brazzaville reported a PCR-corrected efficacy of $97 \%$ for ASAQ and $96.4 \%$ for AL [35]. In the study in Owando et al., the PCR-corrected efficacy was 100\% for ASAQ and 98\% for AL [10].

\section{Plasmodium falciparum in vitro resistance studies}

Two studies have been conducted to assess the level of in vitro resistance of $P$. falciparum parasites to standard anti-malarial drugs. The isotopic test was used in the two studies. The first study was conducted in Brazzaville in 1993 and 34 P. falciparum isolates were tested with chloroquine, quinine and mefloquine. In addition, halofantrine was also tested on 35 P. falciparum isolates. The resistance rates were $61.8,17.7,3$ and $0 \%$ for chloroquine, quinine, mefloquine and halofantrine, respectively [20]. The second investigation was done in Pointe-Noire where P. falciparum isolates were collected from March 2005 to January 2006 and their sensitivity assessed against 11 drugs: chloroquine, quinine, mefloquine, atovaquone, dihydroartemisinin, doxycycline, cycloguanil, lumefantrine, monodesethylamodiaquine, halofantrine and pyrimethamine [29]. The in vitro resistance rates were $75.5 \%$ for chloroquine, $68 \%$ for pyrimethamine, $36 \%$ for cycloguanil, $7 \%$ for mefloquine, $6 \%$ for quinine, $2 \%$ for monodesethylamodiaquine and $0 \%$ for the remaining drugs [29]. These studies contributed demonstrate evidence of high level of chloroquine resistance in the RoC. Since adoption of ACT in 2006, no in vitro study has been conducted on field isolates.

\section{Plasmodium falciparum drug resistance genes}

A total of eight articles have been found reporting data on molecular markers of $P$. falciparum resistance to antimalarials with samples collected from 1999 to 2015 [11, 15, 23-25, 31, 32, 36]. Overall, six molecular markers have been studied: the $P$. falciparum chloroquine resistance transporter ( $p f c r t$ ) gene, associated with chloroquine resistance, the dihydrofolate reductase $(d h f r)$ and the dihydropteroate synthase (dhps) genes, which are linked with pyrimethamine resistance and sulfadoxine resistance, respectively, the P. falciparum Klech-13 (K13) propeller gene, which has recently been linked with artemisinin resistance, and MAL10-688956 and MAL131718319 single nucleotide polymorphisms (SNPs) which have also recently been proposed as molecular markers of artemisinin resistance which is defined as a delayed clearance of $P$. falciparum parasites following ACT. The most analysed molecular markers are pfcrt, dhfr and dhps for which the main findings are summarized in Table 3.
The $p f c r t$ gene has been studied in four articles and for all the K76T mutation was characterized. Nsimba et al. [23] with isolates collected in 1999 at Pointe-Noire and in 2001-2002 at Brazzaville reported a prevalence of 97.1\% of $P$. falciparum isolates carrying the $76 \mathrm{~T}$ mutation. This prevalence was similar to the rate of $98 \%$ reported by Mayengue et al. [24], 88-97\% by Tsumori et al. [11], and $92 \%$ by Koukouikila-Koussounda et al. [15].

Molecular analysis of mutations associated with resistance to antifolates was performed by Nsimba et al. [23], Ndounga et al. [25], Koukouikila-Koussounda et al. [36], and Tsumori et al. [11] (who only analysed the $d h f r$ point mutations). In all of these studies, $d h f r$ point mutations at codons 51, 59 and 108 were found at high frequencies while the mutation at codon 164 was absent. For dhps, high prevalence of the mutation at codon 437 was observed in the three studies, while higher frequency of the mutation at codon 436 and the presence of mutation at codon 540 were only observed in the recent study, with samples collected from pregnant women in 2012-2013 at Brazzaville [36].

The K13 propeller gene was characterized by Mita et al. [32], and MAL10-688956 and MAL13-1718319 SNPs have been characterized by Murai et al. [31]. In both studies they used the same set of archived P. falciparum isolates collected in 2005-2006 in Brazzaville, PointeNoire and Gamboma, and none of the mutations associated with artemisinin resistance was found.

\section{Discussion}

The purpose of this review is to provide an overview of published data and available information on malaria situation in RoC and to identify gaps in knowledge in order to contribute in research-based solutions adapted for the country. In the present review, as the first goal, we attempted to determine if there was any change in malaria epidemiology in RoC from 1992 to 2015, with regard to malaria parasites and vectors and in the presentation of the disease as well. The year 1992 was chosen because the first national policy for malaria control established by the NMCP was adopted that year. Besides, all the research institutions including those involved in malaria research were nationalized.

As a summary, during almost 24 years, a total of 28 studies published in peer reviewed journals were conducted the $\mathrm{RoC}$ in relation to different aspects of malaria infection. Concerning malaria burden, lower rates of malaria parasite infection in children and adults were observed in studies conducted from 2009 to 2015 compared to those conducted from 1992 to 2006 regardless of the diagnostic method (microscopy or PCR). This is suggestive of a decline malaria prevalence and incidence during the past 7 years and might be attributable to the 


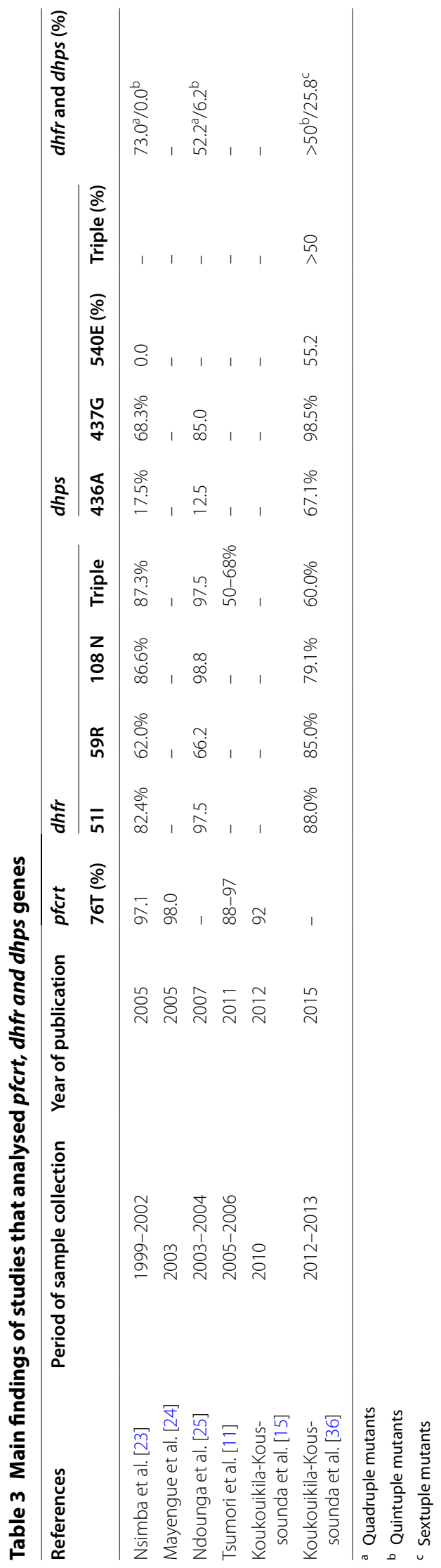


scaling-up of malaria interventions in the country with the support of Global fund for HIV/AIDS, malaria and tuberculosis, including the use ACT which are freely provided to children of $\leq 15$ years in public health facilities since 2008 associated with mass distribution of LLINs from 2008 to 2012. Further evidence on sustainability of the trend to the years later is important to ascertain such attributes.

With regard to Plasmodium species distribution, $P$. falciparum is by far the predominant malaria parasite occurring in the country accounting for almost $100 \%$ of malaria cases [11]. This observation on the extreme predominance of $P$. falciparum in the $\mathrm{RoC}$ is in accordance with findings from studies conducted in other Central African countries [39, 40].

The genetic diversity of $P$. falciparum was characterized trough different studies and the MOI was also determined. These two parameters are known to be good indicators of the level of premunition of populations living in endemic areas and correlate with the extent of the parasite population diversity as well as the transmission intensity $[41,42]$. They are also important determinants of malaria control interventions. Overall, a significant diversity of $P$. falciparum population has been observed in all the studies and this is representative of areas with holo- or hyper-endemic malaria transmission. The High genetic diversity remains similar before and after introduction of ACT in 2006 and scaling up of control measures in the RoC. This implies that most of the parasite clones are still persisting and the interventions did not have impact on specific $P$. falciparum clones. Lower values of the MOI (1.3-1.7) have been obtained in studies conducted after these interventions than those conducted before (2.2-2.3). This trend, in addition to low rates of parasite carriage observed in studies conducted after 2006 may suggest that the implemented control measures have resulted in a substantial decrease of transmission. However, this needs to be assessed by conducting entomological studies that allow determination of the EIR in each area, which correlate well with the level of malaria transmission.

Vector control is one of the main interventions for an effective malaria control programme. To be successful, this intervention needs to rely on availability of data on vector population, level of transmission and insecticide resistance. Unfortunately, only one publication was found [33] and this reflects a major gap in knowledge about the malaria situation in RoC. In that study, 523 An. gambiae complex specimens were collected and the specimen identification revealed that all were An. gambiae s.s., of which, $95.4 \%$ were further molecularly characterized as the S-form. Importantly, these vectors were found to be highly resistant to multiple insecticide classes. This review would classify entomological research as a key priority in the country. Moreove, as An. funestus, An. coustani and An.hancocki were found to be potential minor malaria vectors [5], their implication in malaria transmission and the level of resistance to insecticides have yet to be further elucidated.

Since the utilization of ACT for the treatment of uncomplicated malaria, monitoring studies are required to detect any emergence of artemisinin resistant strains as it has been already reported from South East Asia $[43,44]$. Overall in vivo efficacy studies conducted so far provided evidence of good efficacy of currently recommended ACT in RoC. It is observed that only one study analysed mutations on $P$. falciparum $\mathrm{K} 13$ propeller gene in isolates from $\mathrm{RoC}$ [32], and it would be important that local scientists screen regularly isolates from different parts of the country. Chloroquine and SP were banned for the treatment of uncomplicated malaria in the $\mathrm{RoC}$ since 2006 due to high level of parasite resistance. Studies conducted five to seven years after their withdrawal still show high rates of parasites carrying mutations associated with resistance to these two molecules. This would like to suggest that these molecules remain inactive, despite the fact that SP is still used as intermittent preventive treatment in pregnant women. Moreover, the presence of the $540 \mathrm{dhps}$ mutation (in P. falciparum isolates collected in 2011-2012) [36], which was absent before SP withdrawal [23], and higher level of quintuple dhfr/dhps mutations might suggest that SP resistance become more pronounced.

As a second key priority highlighted by this review is the limited number of publications on malaria in pregnancy in RoC. For instance, there is no data on malaria parasites collected from pregnant women before the introduction of ACT and the first data were published in 2013 [9]. The researchers from Congo working on malaria are also involved in the Central Africa Network on Tuberculosis, HIV/AIDS and Malaria (CANTAM) which aims at building capacities for the conduct of clinical trials. Therefore, it would be of interest to test alternative drugs for preventing malaria during pregnancy in Congolese women.

\section{Conclusion}

A total of 28 peer reviewed articles and two official documents from the NMCP were included in this review and have shown that malaria is still endemic in the country. Unfortunately, the majority of studies were conducted in Brazzaville followed by Pointe-Noire (the two main cities). Therefore, results cannot formally be generalized. We note two major positive points: ASAQ and AL are highly efficacious in treatment of uncomplicated malaria and there is substantial reduction of malaria transmission 
since introduction of ACTs in the country. However, a strong resistance to SP is observed in parasites collected from Congolese pregnant women.

\begin{abstract}
Abbreviations
WHO: World Health Organization; RoC: Republic of Congo; EIR: entomological inoculation rate; ACT: artemisinin-based combination therapy; AL: artemether-lumefantrine; ASAQ: artesunate-amodiaquine; IPTp-SP: intermittent preventive treatment with sulfadoxine-pyrimethamine for pregnant women; LLIN: long-lasting insecticide-treated net; NMCP: National Malaria Control Programme; MHP: Ministry of Health and Population; MeSH: medical subject heading; PCR: polymerase chain reaction; RDT: rapid diagnostic test; msp2: merozoite surface protein 2 gene; msp1: merozoite surface protein 1 GENE; MOI: multiplicity of infection; pfcrt: Plasmodium falciparum chloroquine resistance transporter; dhfr: dihydrofolate reductase; dhps: dihydropteroate synthase; K13: Klech-13; SNP: single nucleotide polymorphism; HIV/AIDS: human immunodeficiency virus/acquired immune deficiency syndrome; CANTAM: Central Africa Network on Tuberculosis, HIV/AIDS and Malaria.
\end{abstract}

\section{Authors' contributions}

KKF carried out the web search, collected documents from the NMCP and drafted the manuscript, NF drafted and reviewed the manuscript. Both authors read and approved the final manuscript.

\section{Author details}

1 Fondation Congolaise Pour la Recherche Médicale, Villa D6, WHO AFRO Campus, Brazzaville, Republic of Congo. ${ }^{2}$ Faculty of Sciences and Techniques, University Marien Ngouabi, Brazzaville, Republic of Congo. ${ }^{3}$ Institute for Tropical Medicine, University of Tübingen, Tübingen, Germany.

\section{Acknowledgements}

Funding was provided by Fondation Congolaise pour la Recherche Medicale (Grant No. 0111).

\section{Competing interests}

The authors declare that they have no competing interests.

Received: 3 September 2016 Accepted: 15 November 2016 Published online: 23 December 2016

\section{References}

1. Autino B, Noris A, Russo R, Castelli F. Epidemiology of malaria in endemic areas. Mediterr J Hematol Infect Dis. 2012;4:e2012060.

2. WHO. World malaria report 2015. Geneva: World Health Organization; 2015. www.who.int/malaria.

3. Cotter C, Sturrock HJW, Hsiang MS, Liu J, Phillips AA, Hwang J, et al. The changing epidemiology of malaria elimination: new strategies for new challenges. Lancet. 2013;382:900-11.

4. Murray CJL, Rosenfeld LC, Lim SS, Andrews KG, Foreman KJ, Haring D, et al. Global malaria mortality between 1980 and 2010: a systematic analysis. Lancet. 2012;379:413-31.

5. NMCP (PNLP). Plan stratégique national de lutte contre le paludisme 2014-2018. Brazzaville. 2014.

6. Samba G, Nganga D, Mpounza M. Rainfall and temperature variations over Congo-Brazzaville between 1950 and 1998. Theor Appl Climatol. 2008;91:85-97.

7. WHO, MHP (Ministère de la Santé et de la Population). Profil entomologique du Congo, rapport technique OMS/Ministère de la Santé et de la Population, Congo 2006.

8. NMCP (PNLP). Rapport d'activités du programme national de lutte contre le paludisme, année 2015. Brazzaville 2016

9. Ntoumi F, Vouvoungui JC, Ibara R, Landry M, Sidibé A. Malaria burden and case management in the Republic of Congo: limited use and application of rapid diagnostic tests results. BMC Public Health. 2013:13:135.
10. Singana BP, Bogreau H, Matondo BD, Dossou-Yovo LR, Casimiro PN, Mbouka R, et al. Malaria burden and anti-malarial drug efficacy in Owando, northern Congo. Malar J. 2016;15:16.

11. Tsumori Y, Ndounga M, Sunahara T, Hayashida $N$, Inoue M, Nakazawa $S$, et al. Plasmodium falciparum: differential selection of drug resistance alleles in contiguous urban and peri-urban areas of Brazzaville, Republic of Congo. PLOS ONE. 2011;6:e23430.

12. Culleton R, Ndounga M, Zeyrek FY, Coban C, Casimiro PN, Takeo S, et al. Evidence of the transmission of Plasmodium vivax in the Republic of Congo, West Central Africa. J Infect Dis. 2009;9:1465-9.

13. Ndounga M, Casimiro PN, Miakassissa-Mpassi V, Loumouamou D, Ntoumi F. Le paludisme dans deux centres de santé au sud de Brazzaville, Congo. Bull Soc Pathol Exot. 2008;101:329-35.

14. Moyen G, Mbika CA, Kambourou J, Oko A, Mouko A. Paludisme grave de l'enfant à Brazzaville. Méd Afr Noire. 2010;57:113-6.

15. Koukouikila-Koussounda F, Malonga V, Mayengue PI, Ndounga M, Vouvoungui CJ, Ntoumi F. Genetic polymorphism of merozoite surface protein 2 and prevalence of K76T pfcrt mutation in Plasmodium falciparum field isolates from Congolese children with asymptomatic infections. Malar J. 2012:11:105.

16. Ntoumi F, Bakoua D, Fesser A, Kombo M, Vouvoungui JC, KoukouikilaKoussounda F. Characterization of asymptomatic Plasmodium falciparum infection and its risk factors in pregnant women from the Republic of Congo. Acta Trop. 2016;153:111-5.

17. Mbongo JA, Ekouya BG, Koulimaya CE, Iloki LH. Paludisme congénital au Centre Hospitalier Universitaire de Brazzaville: une étude épidémiologique de 90 cas. Health Sci Dis. 2015;16:1-5.

18. Ossou-Nguiet PM, Okoko AR, Ekouya BG, Oko AP, Mabiala-Babela JR, Ndjobo MIC, Moyen G. Déterminant du neuropaludisme en milieu pédiatrique Congolais. Revue Neurol. 2013;169:510-4.

19. Oguike MC, Betson M, Burke M, Nolder D, Stothard JR, Kleinschmidt I, et al. Plasmodium ovale curtisi and Plasmodium ovale wallikeri circulate simultaneously in African communities. Int J Parasitol. 2011:41:677-83.

20. Chandenier J, Ndounga M, Carme B, Gay F, Mbitsi A, Hayette MP, et al. Chimiosensibilité in vivo et in vitro de Plasmodium falciparum à Brazzaville (Congo). Cahier Santé. 1995;5:25-9.

21. Durand P, Michalakis Y, Cestier S, Oury B, Leclerc MC, Tibayrenc M, Renaud F. Significant linkage disequilibrium and high genetic diversity in a population of Plasmodium falciparum from an area (Republic of the Congo) highly endemic for malaria. Am J Trop Med Hyg. 2003:68:345-9.

22. Nsimba B, Malonga DA, Mouata AM, Louya F, Kiori J, Malanda M, et al. Efficacy of sulfadoxine/Pyrimethamine in the treatment of uncomplicated Plasmodium falciparum malaria in the Republic of Congo. Am J Trop Med Hyg. 2004;70:133-8.

23. Nsimba B, Jafari-Guemouri S, Malonga DA, Mouata AM, Kiori J, Louya F, et al. Epidemiology of drug-resistant malaria in Republic of Congo: using molecular evidence for monitoring antimalarial drug resistance combined with assessment of antimalarial drug use. Trop Med Int Health. 2005;10:1030-7.

24. Mayengue PI, Ndounga M, Matondo MD, Ntsonde T, Ntoumi F. In vivo chloroquine resistance and prevalence of the pfcrt codon 76 mutation in Plasmodium falciparum isolates from the Republic of Congo. Acta Trop. 2005;95:219-25.

25. Ndounga M, Tahar R, Basco LK, Casimiro PN, Malonga DA, Ntoumi F. Therapeutic efficacy of sulfadoxine-pyrimethamine and the prevalence of molecular markers of resistance in under 5-year olds in Brazzaville, Congo. Trop Med Int Health. 2007;12:1164-71.

26. Van den Broek I, Kitz C, Attas SA, Libama F, Balasegaram M, Guthmann JP. Efficacy of three artemisinin combination therapies for the treatment of uncomplicated Plasmodium falciparum malaria in the Republic of Congo. Malar J. 2006:5:113.

27. Mayengue PI, Ndounga M, Malonga FV, Bitemo M, Ntoumi F. Genetic polymorphism of merozoite surface protein-1 and merozoite surface protein-2 in Plasmodium falciparum isolates from Brazzaville, Republic of Congo. Malar J. 2011;10:276.

28. Ndounga M, Mayengue PI, Casimiro PN, Loumouamou D, Basco LK, Ntoumi F, Brasseur P. Artesunate-amodiaquine efficacy in Congolese children with acute uncomplicated falciparum malaria in Brazzaville. Malar J. 2013;12:53

29. Pradine B, Hovette P, Fusai T, Atanda HL, Baret E, Cheval P, et al. Prevalence of in vitro resistance to eleven standards or new antimalarial drugs 
among Plasmodium falciparum isolates from Pointe-Noire, Republic of the Congo. J Clin Microbiol. 2006;44:2404-8.

30. Ndounga M, Tahar R, Casimiro PN, Loumouamou D, Basco LK. Clinical efficacy of artemether-lumefantrine in Congolese children with acute uncomplicated falciparum malria in Brazzaville. Malar Res Treat. 2012;2012:749479.

31. Murai K, Culleton R, Hisaoka T, Endo H, Mita T. Global distribution of polymorphisms associated with delayed Plasmodium falciparum parasite clearance following artemisinin treatment: genotyping of archive blood samples. Parasitol Int. 2015;64:267-73.

32. Mita T, Culleton R, Takahashi N, Nakamura M, Tsukahara T, Hunja CW, et al. Little polymorphism at the K13 propeller locus in worldwide Plasmodium falciparum populations prior to the introduction of artemisinin combination therapies. Antimicrob Agents Chemother. 2016;60:3340-7.

33. Koekemoer LL, Spillings BL, Christian RN, Lo TCM, Kaiser ML, Norton RA, et al. Multiple insecticide resistance in Anopheles gambiae (Diptera: Culicidae) from Pointe Noire, Republic of the Congo. Vector Borne Zoonotic Dis. 2011:11:1193-200.

34. Ibara-Okabande R, Koukouikila-Koussounda F, Ndounga M, Vouvoungui J, Malongua V, Casimiro PN, et al. Reduction of multiplicity of infections but no changes in msp2 genetic diversity in Plasmodium falciparum isolates from Congolese children after introduction of artemisinin-combination therapy. Malar J. 2012;11:410.

35. Ndounga M, Mayengue PI, Casimiro PN, Koukouikila-Koussounda F, Bitemo M, Matondo BD, et al. Artesunate-amodiaquine versus artemether-lumefantrine for the treatment of acute uncomplicated malaria in Congolese children under 10 years old living in suburban area: a randomized study. Malar J. 2015;14:423.
36. Koukouikila-Koussounda F, Bakoua D, Fesser A, Nkombo M, Vouvoungui C, Ntoumi F. High prevalence of sulphadoxine-pyrimethamine resistanceassociated mutations in Plasmodium falciparum field isolates from pregnant women in Brazzaville, Republic of Congo. Infect Genetics Evol. 2015;33:32-6.

37. Trape JF, Zoulani A. Malaria and urbanization in central Africa: the example of Brazzaville. Part II: results of entomological surveys and epidemiological analysis. Trans R Soc Trop Med Hyg. 1987;81(suppl 2):10-8.

38. MHP (Ministère de la Santé et de la Population). Politique nationale de lutte contre le paludisme, Brazzaville, République du Congo 2006.

39. Fru-Cho J, Bumah VV, Safeukui I, Nkuo-Akenji T, Titanji VP, HIdar K. Molecular typing reveals substantial Plasmodium vivax infection in asymptomatic adults in rural area of Cameroon. Malar J. 2014;13:170.

40. Culleton RL, Mita T, Ndounga M, Unger H, Cravo PV, Paganotti GM, et al. Failure to detect Plasmodium vivax in West and Central Africa by PCR species typing. Malar J. 2008;7:174.

41. Babiker HA, Ranford-Cartwright LC, Walliker D. Genetic structure and dynamics of Plasmodium falciparum infections in the Kilombero region of Tanzania. Trans R Soc Trop Med Hyg. 1999;93:11-4.

42. Smith T, Beck HP, Kitua A, Mwankusye S, Feldrl I, Fraser-Hurt N, et al. Epidemiology of multiple Plasmodium falciparum infections. Age dependence of the multiplicity of Plasmodium falciparum infections and other malariological indices in an area of high endemicity. Trans R Soc Trop Med Hyg. 1999:93:15-20.

43. Noedl H, Se Y, Schaecher K, Smith BL, Socheat D, Fukuda MM. Evidence of artemisinin-resistant malaria in western Cambodia. N Engl J Med. 2008;359:2619-20.

44. Dondorp AM, Nosten F, Yi P, Das D, Phyo AP, Tarning J, et al. Artemisinin resistance in Plasmodium falciparum malaria. N Engl J Med. 2009;361:455-67.

\section{Submit your next manuscript to BioMed Central and we will help you at every step:}

- We accept pre-submission inquiries

- Our selector tool helps you to find the most relevant journal

- We provide round the clock customer support

- Convenient online submission

- Thorough peer review

- Inclusion in PubMed and all major indexing services

- Maximum visibility for your research

Submit your manuscript at www.biomedcentral.com/submit 